\title{
La enseñanza de español como lengua de herencia en Estados Unidos: razones socioeconómicas y educativas que justifican su docencia universitaria en la década de 2020
}

\author{
TEACHING SPANISH AS A HERITAGE LANGUAGE IN THE UNITED STATES: \\ SOCIOECONOMIC AND EDUCATIONAL REASONS THAT JUSTIFY ITS UNIVERSITY \\ INSTRUCTION IN THE 2020 S
}

\section{Erik Garabaya Casado \\ Universidad de Oviedo / University of Utah}

Resumen

\begin{abstract}
La pedagogía del español como lengua de herencia es una disciplina académica con cada vez mayor demanda universitaria en Estados Unidos. Esto se debe a una serie de motivos sociales, económicos y educativos que presentan una realidad nacional muy distinta a la que imperaba a finales del siglo pasado. En este artículo repasamos algunas de las razones que consideramos más relevantes, como lo son el aumento demográfico de la población hispana, la mejora de su poder adquisitivo, una mayor aceptación social en lo referente al bilingüismo y un gran desarrollo en la investigación y docencia de las lenguas de herencia. En una coyuntura en la que la comunidad hispanoestadounidense ha sobrepasado los 60 millones de personas, ha superado el PIB de España y que está más presente que nunca en la educación superior, resulta indispensable promover una instrucción universitaria dirigida a recuperar la competencia en castellano de este perfil de hablantes si queremos mantener y potenciar su desarrollo lingüístico durante los próximos diez años.
\end{abstract}

Palabras Clave: español lengua de herencia, hablantes de herencia, sociolingüística, pedagogía del lenguaje, español en Estados Unidos
Abstract

The pedagogy of Spanish as a heritage language is an academic discipline with increasing demand at the universities in the United States. This is due to a series of social, economic, and educational reasons that present a national reality quite different from the one that prevailed at the end of the last century. This article reviews some of the most relevant causes, such as the demographic increase of the Hispanic population, the improvement of their purchasing power, a greater social acceptance regarding bilingualism, and a deep development in the research and teaching of heritage languages. In a situation in which the Hispanic community has exceeded 60 million people, surpassed Spain's GDP, and is more present than ever in higher education, it is essential to promote university instruction aimed at recovering competence in Spanish of this profile of speakers if we want to maintain and enhance their linguistic development during the next ten years.

KEY WORDS: Spanish heritage language, heritage speakers, sociolinguistics, language pedagogy, Spanish in the United States 


\section{INTRODUCCIÓN}

La docencia del español como lengua de herencia es un campo que se ha ido especializando y asentando en las últimas dos décadas. Este tipo de enseñanza, dirigida a un perfil de alumnado muy específico por tener ya cierta competencia lingüística y cultural en la lengua castellana, se está convirtiendo en una necesidad curricular a medida que el número de alumnos potenciales aumenta debido a diversas cuestiones demográficas y económicas.

Este artículo pretende recoger algunas de las causas que consideramos más relevantes a la hora de justificar la aparición de cursos de herencia en la educación superior. En el primer apartado, resumiremos los atributos más esenciales que caracterizan al hablante de herencia y que lo contraponen al alumnado de español para nativos y al de español como segunda lengua o lengua extranjera. En segundo lugar, analizaremos el estado socioeconómico que atraviesa la población hispana en el contexto estadounidense actual, desde su más que evidente crecimiento demográfico hasta la mejora en su poder adquisitivo, la cual beneficia la presencia de estudiantes de herencia en las universidades. La consolidación de la clase media hispana, aún inestable como ha quedado patente tras el impacto económico sufrido a causa de la COVID-19, permite un mayor acceso a la educación postobligatoria y, con ello, a los cursos a los que nos estamos refiriendo. Por último, comentaremos la apertura de la sociedad estadounidense hacia el bilingüismo, tanto por parte de los grupos sociales dominantes como por los minoritarios, que podemos ver reflejado en el creciente éxito de los programas de inmersión dual primaria y que presenta un escenario mucho más propicio para desarrollar programas bilingües que se ajusten a los nuevos intereses de la nación.

Todo ello viene auspiciado por una situación en la que el estudio teórico y la experimentación práctica en lo relativo a las lenguas de herencia cuenta con más personal formado que nunca. La labor conjunta de los investigadores, comunicadores, profesores, departamentos, organizadores de conferencias, cursos y talleres, y diseñadores de materiales ha consolidado una pedagogía del lenguaje singular, cada vez más necesaria en el gigante norteamericano, como nos vamos a encargar de argumentar a continuación.

\section{DEFINIENDO AL ESTUDIANTE DE LENGUA DE HERENCIA}

La enseñanza de español a aquellos individuos estadounidenses que, en principio, pueden considerarse parcialmente como «hispanohablantes» se remonta al siglo XIX y a la incorporación de los antiguos estados mexicanos del suroeste a la Unión (Wright, 2019: 70). Sin embargo, el término «lengua de herencia» que manejamos hoy no se populariza hasta finales de los 60, asociado a varios programas educativos canadienses que trataron de visibilizar el multiculturalismo étnico de sus ciudadanos (García, 2009: cap. 3). A finales del siglo XX, se adopta este término también en el ámbito estadounidense, dirigido a los hablantes de las lenguas distintas al inglés, incluyendo las lenguas nativas americanas (Valdés, 2001: 38-39). Hasta entonces, ya había cursos para enseñar el español a hijos de inmigrantes, pero estos normalmente se agrupaban bajo denominaciones como español «para nativos» O «para bilingües», las cuales no eran tan precisas a la hora de reflejar las 
La enseñanza de español como lengua de herencia en Estados Unidos: razones socioeconómicas y educativas que justifican su docencia universitaria en la década de 2020 | E. Garabaya Casado

necesidades pedagógicas y lingüísticas de estos aprendientes (Acosta Corte, 2013; SaidMohand, 2013; Valdés, 2001).

La definición más citada hasta la fecha para referirse a los «hablante de herencia» fue propuesta por Valdés (2000: 1), quien designa a estos individuos como aquellos que crecen en un hogar donde se habla un idioma distinto al inglés, que son capaces de comunicarse en este o solamente de entenderlo, y que pueden considerarse bilingües en este idioma y en inglés hasta cierto punto. Si bien su lengua de herencia es hablada en el hogar, la educación formal que reciben desde la infancia es en inglés, por lo que generalmente se terminan alfabetizando solo en la lengua dominante. Puesto que la adquisición del primer idioma se detiene para dejar paso a uno nuevo, también es frecuente encontrar el término de «bilingües secuenciales» (Montrul, 2008: 162; Silva-Corvalán, 2012: 783) referido a estos hablantes.

Estas circunstancias socioeducativas empujan al individuo a una vía de adquisición idiomática que difiere enormemente de las de los monolingües en español. Una menor exposición a la lengua minoritaria y menos oportunidades de usarla conlleva diferencias cada vez más pronunciadas respecto a la lengua de la generación anterior, lo que perpetúa una reducción del sistema gramatical y una menor competencia de tipo pragmáticodiscursivo (Silva-Corvalán, 2014, 2018). Potowski et alii (2009) enumeran tres fenómenos, no necesariamente simultáneos, que son clave a la hora de entender el español que manejan los hablantes de herencia: la adquisición incompleta, el desgaste y la adquisición de una variedad de contacto.

En primer lugar, se produce una adquisición incompleta en la medida que la irrupción del inglés reduce repentinamente la exposición lingüística del niño al castellano y dificulta o interrumpe su adquisición. Esto suele ocurrir cuando el niño que está aprendiendo la lengua minoritaria en el hogar comienza la instrucción académica por completo en inglés, normalmente entre los 4 o 5 años, e impide la adquisición de elementos propios de estadios lingüísticos más avanzados' (Acosta Corte, 2013). Por lo tanto, cuanto menor sea el niño en el momento en el que esta interrupción suceda debido a la aparición de la nueva lengua, menos desarrollada será su competencia en español en su etapa adulta (Montrul, 2002: 61, 2008: 161).

El segundo fenómeno se ha denominado desgaste (attrition). Este hecho se refiere al proceso por el cual se pierde un aspecto del sistema lingüístico que ya había sido adquirido completamente (Montrul, 2002: 40; Potowski et alii, 2009: 538). Normalmente, es la poca frecuencia en su uso y de exposición a la lengua la que provoca una especie de «olvido» 0 pérdida del idioma. No obstante, resulta extraordinariamente complicado determinar cuál de los dos fenómenos comentados hasta ahora ha ocurrido en el desarrollo lingüístico del

\footnotetext{
1 Uno de los ejemplos más citados para ilustrar esta problemática es el de la adquisición del modo subjuntivo (Potowski et alii, 2009). El estudio realizado por Blake (1983) con monolingües en español sugiere la existencia de un «periodo de transición» entre los 5 y 8 años en el que el niño muestra irregularidades en la producción de oraciones correctas en subjuntivo, una situación que no se estabiliza hasta los 10-12 años. Esto nos puede dar una idea del impacto que puede causar la adquisición incompleta del español en los bilingües secuenciales de Estados Unidos, en la medida en que esta suele producirse durante o incluso antes de llegar a dichas edades.
} 
La enseñanza de español como lengua de herencia en Estados Unidos: razones socioeconómicas y educativas que justifican su docencia universitaria en la década de 2020 | E. Garabaya Casado

hablante (Montrul, 2008: 164), por lo que, para poder distinguir entre ambos, es imprescindible la elaboración de estudios longitudinales que clarifiquen qué elementos llegaron a ser adquiridos y cuáles no (Potowski et alii, 2009: 538).

Por último, hay que tener en cuenta que muchos estudiantes han adquirido una variedad de contacto. Se entiende por esta como la adquisición completa de una variedad del castellano influida por el contexto bilingüe en el que viven sus hablantes (Potowski et alii, 2009: 539). De ahí que estos estudiantes acudan al aula no solo con el vocabulario propio de los distintos dialectos hispánicos de sus familiares (v. Lipski, 2015), sino también con una variedad altamente influenciada por el inglés. Algunos de los rasgos más característicos en lo relativo a esto último son los préstamos, los calcos, las extensiones semánticas o el cambio de código (Potowski, 2005: 25).

Esta triple fenomenología, sumada a la situación sociocultural que ya de por sí atraviesan los hispanos en este país, singulariza el perfil del estudiante de herencia. Dependiendo de en qué medida afecten estos eventos al individuo, podemos encontrarnos un rango de competencia lingüística muy dispar, oscilando dentro de un amplio espectro o "continuo de bilingüismo" (Silva-Corvalán, 2012, 2018; Valdés, 2001). La vasta heterogeneidad de sus aprendientes es uno de los motivos que más complican la elaboración de una pedagogía específica que se ajuste a sus necesidades (Fuller y Leeman, 2020: 211; Schwartz, 2001: 237). Debido a esto, resulta pertinente que los cursos de herencia incorporen al inicio del semestre la elaboración de pruebas diagnósticas que evalúen el nivel idiomático de cada estudiante (Potowski, 2005: 65-66; Said-Mohand, 2013), así como encuestas que recojan información de su trasfondo familiar y cultural (Carreira y Kagan, 2011), con el propósito de adaptar la materia lo más posible a los participantes.

Por lo general, los estudiantes que buscan matricularse en cursos de herencia lo hacen atendiendo a unas motivaciones particulares. Estas suelen dirigirse, por una parte, a razones de tipo afectivo y cultural, a una «motivación de herencia» (Van Deusen-Scholl, 2003: 222) de reconectar con sus raíces, lo que suele exigir a los instructores que demuestren cierta sensibilidad y empatía con la clase de experiencias de vida que han atravesado los alumnos. El segundo tipo de motivación, muchas veces complementaria a la anterior, son las razones económicas y profesionales. A este respecto, es preciso destacar que los hablantes de herencia han experimentado la lengua solo en contextos familiares, informales y orales-auditivos (Montrul, 2008: 164-167), por lo que el contenido curricular debe enfocarse en enseñar un lenguaje académico, formal y escrito en español al que los alumnos no han tenido acceso por escolarizarse en inglés.

Como hemos visto hasta ahora, los hablantes de herencia no desarrollan una competencia lingüística equiparable a la de los hablantes nativos o monolingües en español, y de ahí que no deban ser colocados en cursos de lengua dirigidos a estos últimos. No obstante, su competencia limitada y un trasfondo familiar y cultural ligado al mundo hispánico también les distancia de los estudiantes de español como segunda lengua o lengua extranjera (ELE), quienes suelen empezar desde un desconocimiento absoluto y aprenden el idioma solo en el entorno académico. Por este motivo, son muchos los autores que claman también por 
La enseñanza de español como lengua de herencia en Estados Unidos: razones socioeconómicas y educativas que justifican su docencia universitaria en la década de 2020 | E. Garabaya Casado

una separación entre los alumnos de ELE y los de lengua de herencia en los programas de idiomas (p. ej., Acosta Corte, 2013; García, 2009; Potowski, 2005).

Este ha sido un breve repaso de las circunstancias sociolingüísticas por las que transita el estudiante de herencia antes de llegar al aula universitaria. A continuación, ahondaremos en el aspecto socioeconómico, el cual creemos imprescindible para explicar las causas que moldean la realidad lingüística de los aprendientes y la forma en la que interaccionan con ella y con el resto de la comunidad de hablantes.

\section{EL CAMBIO SOCIOECONÓMICO EN LA ÚLTIMA DÉCADA}

El análisis económico ligado al desarrollo de la lengua española en el mundo suele gravitar en torno a la atracción irresistible de los Estados Unidos. Prueba de ello es que instituciones académicas tan importantes como el Instituto Cervantes hayan puesto sus ojos en el gigante norteamericano y dediquen amplios apartados de su anuario El español: una lengua viva a divulgar su crecimiento en dicho territorio (véase, por ejemplo, Instituto Cervantes, 2020).

Sin embargo, el grueso de estos análisis apunta normalmente a la expansión del español como lengua extranjera, es decir, la que se enseña a los que son hablantes nativos de inglés. En este artículo, hemos querido separarnos de esta disciplina para concentrarnos exclusivamente en sus cifras respecto a la lengua de herencia y a los hispanohablantes. $Y$ es que, si bien los datos relativos a las matriculaciones de estudiantes no hispanos generalmente anglosajones - , en cursos de español son más que positivos², los números respecto a los hablantes de herencia requieren otro tipo de exploración.

Por ello, algunos de los hitos que examinaremos en este apartado tienen que ver con el reciente sobrepaso de los 60 millones de individuos catalogados como "hispanos» o «latinos» en el censo estadounidense (Instituto Cervantes, 2020: 43; U. S. Census Bureau, 2020) y las consecuencias asociadas a este crecimiento poblacional, así como el aumento de su poder adquisitivo, que les ha permitido empezar a consolidar una clase media relativamente estable.

\subsection{Sesenta millones de «hispanos»}

La década de 2010 cerró con una cifra destacada. La comunidad hispana superó los 60 millones de habitantes en este país en 2019 , más del $18 \%$ del global, eso sin contar a las personas indocumentadas. Por ubicarlo dentro de una perspectiva histórica, la anterior fecha de referencia fue el año 2000, en el que la población hispana había alcanzado el $13 \%$ y se había establecido así como el grupo minoritario más amplio (Potowski, 2005: 11), por encima del afroamericano.

\footnotetext{
2 Las estimaciones del Instituto Cervantes (2020: 13) apuntan a que alrededor de 8 millones de estudiantes estadounidenses de todas las edades escogen aprender español. De ellos, más de 700000 están en la enseñanza universitaria, una cifra que supera a la suma de matriculados en el resto de las lenguas extranjeras juntas (Instituto Cervantes, 2020: 54).
} 
La enseñanza de español como lengua de herencia en Estados Unidos: razones socioeconómicas y educativas que justifican su docencia universitaria en la década de 2020 | E. Garabaya Casado

No obstante, el hecho de ser «hispano» no convierte a uno automáticamente en «hispanohablante». De hecho, todavía a día de hoy es difícil catalogar a individuos como «hablantes» de español en una situación de bilingüismo tan compleja como esta. El Instituto Cervantes (2020) ha tratado de unificar el criterio a este respecto en los últimos años diferenciando entre "grupo de dominio nativo» y "grupo de competencia limitada». De acuerdo con esta división, Estados Unidos pasaría de tener cerca de 56 millones de hispanohablantes potenciales a alrededor de 41 millones de hablantes «nativos» y 15 millones de hablantes con "competencia limitada» (Instituto Cervantes, 2020: 9), ateniéndonos a la nomenclatura propuesta. Esto coloca al país en el quinto lugar mundial -y no en el segundo, como se había asegurado hace unos años - , por detrás de México (124 millones), Colombia (50), España (47) y Argentina (45), en cuanto al número de hablantes con dominio nativo (Instituto Cervantes, 2020: 7).

A estas dos categorías de hablantes cabría sumar una tercera alternativa, referida a los individuos étnicamente censados como «hispanos» o «latinos» en Estados Unidos -el censo estadounidense utiliza ambos términos indistintamente-, pero que carecen de una competencia en español suficiente para integrarse en ninguna de las dos posibilidades de hablante, o que directamente desconocen la lengua. Si contrastamos los 56 millones de hablantes potenciales recogidos por el Cervantes con los 60 millones de personas pertenecientes a este grupo étnico censado por la administración, nos encontraríamos con que cerca de 4 millones de hispanos no serían, al mismo tiempo, hispanohablantes; eso sin contar con que no estaríamos considerando en el cálculo a los individuos anglosajones o de otras etnias minoritarias que sí lo hayan aprendido.

Como se viene alertando desde hace más de una década, el factor que explica que el número de hispanohablantes esté creciendo en el país es la constante llegada de inmigrantes que ya han adquirido el castellano como primera lengua en sus países de origen, y no que estos estén transmitiendo su idioma a sus descendientes (Carreira, 2007: 539; Lipski, 2015: 363-364; Potowski, 2005: 16). El patrón habitual de transferencia lingüística es que la primera generación de hablantes sea monolingüe en español, la segunda generación - nacida ya en el nuevo país o llegada durante la infancia - sea hasta cierto punto bilingüe, con el inglés como idioma dominante, y la tercera generación sea ya monolingüe en inglés ${ }^{3}$ (Montrul, 2008: 162-163; Silva-Corvalán, 2014: 2; cf. Valdés, 2001: 43). A esto se lo ha denominado "sustitución lingüística» o language shift, y es una circunstancia que se ha repetido con otras lenguas de inmigración del pasado (García, 2009: cap. 4). De hecho, en el caso de la comunidad hispanohablante, este efecto se estaría produciendo más rápido que nunca (Potowski, 2005: 30).

Esto implica una serie de problemas que, a nuestro juicio, dejan al español en una situación preocupante. Por una parte, impide la consolidación de un dialecto propiamente estadounidense (v. Moreno Fernández, 2008) al que estos hablantes se puedan aferrar; por otra, propicia un triunfalismo y mercantilización del español que se basan en una

${ }^{3}$ El Instituto Cervantes (2020: 50) recoge que el $61 \%$ de los hispanohablantes adultos de primera generación declaran tener el español como su lengua principal, algo que decrece dramáticamente en la segunda generación (8 \%) y más aún en la tercera y siguientes (1\%). 
La enseñanza de español como lengua de herencia en Estados Unidos: razones socioeconómicas y educativas que justifican su docencia universitaria en la década de 2020 | E. Garabaya Casado

imagen azucarada de la situación lingüística real (Fuller y Leeman, 2020: 258) y evita que se tomen las medidas necesarias; $y$, sobre todo, genera una brecha generacional por la que padres y abuelos no pueden comunicarse con sus descendientes, lo que impide la transmisión de conocimiento, valores y cultura, y origina un problema de identidad para las nuevas generaciones (Wong Fillmore, 1991).

Enfrentar estas adversidades pasa precisamente por la promoción de una enseñanza de lengua de herencia que «recupere» a todos estos hablantes potenciales apelando a unos lazos familiares y culturales que ya poseen. Sin embargo, la realidad indica que la prosperidad económica en buena parte del país ha solido demandar que los hispanohablantes sacrifiquen su español (Lipski, 2003: 233-234). Tratar de revertir esta dinámica a través de la educación ha de plantearse como un objetivo fundamental.

Para 2030, se calcula que uno de cada cinco estadounidenses sea de origen hispano (Joint Economic Committee, 2020: 6). El Instituto Cervantes (2020: 16-17) va más allá y sostiene que para 2060 un tercio de la población estadounidense podría pertenecer ya a esta etnia, casi 111 millones de personas distribuidas por todo el territorio. Está en las manos de la educación bilingüe materializar que este crecimiento demográfico se traduzca también en un crecimiento de la lengua. Como siempre, esto implica dotar de prestigio al castellano dentro de sus fronteras, que deje de percibirse como un idioma de inmigración y fracaso educativo y económico (Fishman, 2001: 92), especialmente desde el seno familiar, y divulgar las bondades del bilingüismo y biculturalismo a la hora de constituir una sociedad más productiva y tolerante. Quizá esto pase por desechar la idea clásica del «crisol» estadounidense (melting pot), una metáfora que, en realidad, alude al combate contra el multilingüismo y a una asimilación de las minorías dentro de la cultura mayoritaria (Van Deusen-Scholl, 2003: 215), y empezar a promover la del «bol de ensalada» (Wright, 2019: 21), en la que, por el contrario, cada ingrediente mantiene sus cualidades individuales para aportar un color especial al conjunto.

\subsection{El desarrollo de una clase media hispana}

El aumento del número de hispanos, como vimos, no supone por sí solo una mejora del escenario lingüístico del territorio. Tal como afirma Carreira (2007: 522), el valor económico del español no radica únicamente en el número de hablantes, sino también en otros factores como la condición económica de estos, la extensión geográfica que abarquen, su repercusión en los medios culturales y el uso de la lengua en los sectores público y privado.

En este aspecto, la situación financiera de los hispanohablantes estadounidenses ha mejorado notablemente. Prueba de ello es que, en lo relativo al PIB generado dentro del registro nacional, la comunidad hispana de Estados Unidos es el grupo étnico que más rápido está creciendo económicamente, y ya sería considerada la octava economía del mundo si se tratase de un país independiente, superando a España y a todos los países latinoamericanos (Instituto Cervantes, 2020: 23-24). Por consiguiente, resulta indudable que la población latina se está consolidando dentro de la clase media estadounidense (Jiménez, 2019: 149). 
La enseñanza de español como lengua de herencia en Estados Unidos: razones socioeconómicas y educativas que justifican su docencia universitaria en la década de 2020 | E. Garabaya Casado

Con todo, esta comunidad es aún muy vulnerable ante las adversidades socioeconómicas que puedan agitar al país. Por ejemplo, López et alii (2020) documentan que la población hispana, junto a la afroamericana, fue la más afectada por la pandemia de COVID-19. Este colectivo soportó unas dificultades más acentuadas que la población blanca a la hora de hacer frente a las pérdidas de salario o trabajo, o de pagar las facturas mensuales.

Según un reporte del Congreso estadounidense (Joint Economic Committee, 2020), 40000 individuos hispanos fallecieron en los seis primeros meses de la emergencia sanitaria. De hecho, los datos indican que tuvieron el doble de posibilidades de perder la vida a causa del virus que la comunidad blanca. A esto hay que añadir que más de tres de cada cinco hogares hispanos sufrieron una reducción del salario laboral, perdieron el acceso a la cobertura sanitaria y experimentaron inseguridad alimenticia. Además, su tasa de desempleo subió de un 6 \% a un 18,9 \% en tan solo un mes (marzo-abril de 2020), lo que afectó a 5 millones de trabajadores. Paradójicamente, los que sí mantuvieron el empleo fueron los más vulnerables a contraer el virus al pertenecer a puestos de trabajo esenciales cuya actividad no podía detenerse ni realizarse desde casa. Seis meses después del estallido de la pandemia, el desempleo hispano aún se mantenía en el 10,5 \%.

Ante este tipo de escenarios tan impredecibles, la universidad debe erigirse como una garantía que permita a los estudiantes conservar o mejorar sus expectativas de vida. Promocionar el carácter instrumental del lenguaje, además del afectivo, puede ser una de las principales estrategias para los cursos de idiomas. Es decir, es indispensable que los hablantes la perciban económicamente como una «lengua útil» (Jiménez, 2019: 154).

Carreira y Armengol (2001: 109) señalan que la expansión del mercado globalizado beneficia la enseñanza del español, puesto que ha hecho que conocer distintas lenguas aparte del inglés sea ya más una necesidad que un lujo. Algunos autores se han encargado de cuantificar los beneficios económicos que puede reportar el bilingüismo en Estados Unidos. Agirdag (2014: 177) estima que los bilingües balanceados -aquellos hispanos que tienen una alta competencia en los dos idiomas - tienen mayores posibilidades de encontrar un trabajo y que este sea a tiempo completo que aquellos hablantes que tienen el inglés como lengua dominante. Por otro lado, los primeros tienen un salario superior, que normalmente oscila entre los 2000 y 3200 dólares anuales por encima del de los segundos.

Esto no ha pasado desapercibido para la población estudiantil hispana y ha propiciado que el aspecto económico sea uno de los mayores reclamos. En unas encuestas formuladas a estudiantes de herencia universitarios en Nuevo México, Hudgens Henderson et alii (2020) hallaron que los alumnos estimaban más positivamente el factor instrumental del idioma que estaban aprendiendo por encima de otras variables como el aspecto sentimental, el comunicativo o el valor identitario. Estas conclusiones coinciden con las realizadas por Carreira y Kagan (2011), donde los alumnos de español valoraban los objetivos laborales por encima del resto de factores, algo no necesariamente correspondido en el caso de aprendientes de otras lenguas minoritarias. No obstante, cuanto mayor es el nivel del curso de español y, por ende, mejor pueden comunicarse en este idioma, mejor valoran la lengua 
La enseñanza de español como lengua de herencia en Estados Unidos: razones socioeconómicas y educativas que justifican su docencia universitaria en la década de 2020 | E. Garabaya Casado

y demuestran un mayor deseo de hablarla más allá de cuestiones tan utilitarias (Hudgens Henderson et alii, 2020: 40).

En resumen, tanto la incesante crecida demográfica como el incremento del poder adquisitivo se traducen en una mayor demanda para acceder a la educación superior y, en consecuencia, para matricularse en cursos de español. El aumento de estudiantes que solicitan participar en programas de herencia en las universidades no deja de crecer (Fuller y Leeman, 2020: 266; Hudgens Henderson et alii, 2020: 27), lo que arroja una perspectiva muy positiva para el desarrollo de este campo y de la enseñanza de lenguas en general. Los docentes deberán encargarse de conciliar las distintas necesidades y motivaciones de sus alumnos, que pueden ir desde razones emocionales y de búsqueda de la identidad personal, hasta motivos puramente pragmáticos y económicos, o ambos extremos al mismo tiempo.

La educación bilingüe supone, a nuestro juicio, un sostén de vital importancia para la conservación de la rica variedad lingüística en suelo estadounidense, más allá del español. En el último apartado, haremos un repaso de dos de los tipos de programas bilingües que más impacto han tenido en los últimos diez años: la inmersión dual y la propia enseñanza de herencia (Fuller y Leeman, 2020: 265).

4 PERFECCIONAMIENTO DE LA PEDAGOGÍA ESPECÍFICA DE LENGUAS DE HERENCIA Y DEMANDA DE BILINGÜISMO

\subsection{El «boom» de la inmersión dual}

La creciente aceptación del bilingüismo por parte de la sociedad estadounidense está favoreciendo la aparición de programas de lenguas en todo el territorio. Sin embargo, esto no siempre ha sido así. En el año 2002, la promulgación del acta No Child Left Behind por parte de la administración de George W. Bush - una política educativa que derogó la anterior Bilingual Education Act, aprobada en el contexto histórico de la lucha por los derechos civiles-, comprometió los avances en materia de promoción de segundas lenguas. Por una parte, la medida relegó las clases de lenguas extranjeras a un segundo plano, puesto que los exámenes oficiales se centraban solo en contenido académico relativo a las matemáticas, ciencias, ciencias sociales o lengua inglesa, bajo riesgo de sanción económica para las instituciones escolares que no alcanzasen los objetivos fijados (Carreira, 2007: 540; Menken, 2010: 122). Por otra, eliminó directamente cualquier mención oficial al «bilingüismo» (Menken, 2010: 122; Wright, 2019: 75) y forzó a los niños inmigrantes a renunciar a su lengua materna para realizar una rápida transición al inglés (Baker y Wright, 2017: 373), con los perjuicios cognitivos, académicos y sociales que esto conlleva (v. Collier y Thomas, 2007). Todo ello promovido por un clima social con claros tintes xenófobos de la mano del movimiento English Only, cuyos partidarios argumentan que el monolingüismo en inglés contribuye a la homogeneización de los extranjeros dentro del ideal estadounidense y que el multilingüismo es problemático y debe rechazarse (Baker y Wright, 2017: cap. 17; Dicker, 2000). 
La enseñanza de español como lengua de herencia en Estados Unidos: razones socioeconómicas y educativas que justifican su docencia universitaria en la década de 2020 | E. Garabaya Casado

La situación se ha ido invirtiendo en las dos décadas posteriores, y hoy en día los programas de inmersión dual puestos en práctica en cada vez más escuelas primarias del país son un buen ejemplo de la mejoría. Estos consisten en impartir los contenidos académicos tanto en inglés como en la lengua minoritaria, repartiendo el tiempo de instrucción equitativamente o favoreciendo a esta última; por ejemplo, matemáticas o biología en español. Algunas de las ventajas de este modelo consisten en evitar la segregación en las aulas - ya que se pueden aprovechar de él tanto niños de origen anglosajón como inmigrante-, enseñar dos idiomas de manera contextualizada mientras se avanza en los contenidos escolares, instruir a los aprendientes en un registro formal al que no están expuestos en sus hogares y, sobre todo, demostrar valor y respeto por las lenguas maternas que adquieren los niños de sus familias al situarlas al mismo nivel comunicativo que el inglés (Baker y Wright, 2017; Collier y Thomas, 2007; Wright, 2019).

Sin embargo, la educación bilingüe debe prolongarse en el tiempo si aspira a ser efectiva. Uno de los ejemplos más actuales es la iniciativa puesta en marcha en Utah, que ha supuesto un «boom» imitado por más de 30 estados (Valdez et alii, 2016: 604; Wright, 2019: 110). Watzinger-Tharp et alii (2021: 200-201) enumeran las distintas fases en las que se articula el sistema educativo bilingüe en Utah hasta llegar al nivel universitario. En primer lugar, la ya comentada inmersión dual comienza en el kindergarten (5-6 años) o primer grado de la escuela primaria (6-7 años) hasta quinto grado (10-11 años). En este periodo, los niños atienden a una escolarización 50-50 en inglés y en la lengua meta, y la disposición en el aula puede ser tanto de una vía (one-way) - niños procedentes de un único grupo étnico, normalmente del anglosajón - como de doble vía (two-way)-balance de niños de ambas lenguas maternas - ${ }^{4}$. En segundo lugar, los estudiantes continúan con el aprendizaje de la segunda lengua en la escuela intermedia (middle school, entre los 11 y 14 años) con un curso de lengua extranjera y otro sobre cultura, historia y medios de comunicación en el segundo idioma (30 \% del tiempo en la lengua meta). Por último, en la escuela superior (high school, sobre los 14 a 18 años), los adolescentes pueden participar en un programa puente o Bridge Program mediante el cual atienden a un curso anual en la lengua meta canjeable por créditos universitarios (menos del $20 \%$ del tiempo). La nota negativa es que, como podemos ver, si bien el alumno puede mantener el contacto con la lengua elegida durante todo el periodo escolar, el porcentaje de exposición a esta va decreciendo conforme se supera cada nivel educativo.

La popularidad incipiente de los itinerarios bilingües o duales en el sistema escolar puede tener una gran repercusión en los programas de lengua de herencia para adultos. Por una parte, porque, tal y como indican Carreira y Kagan (2018: 163), constituyen un referente a la hora de institucionalizar la enseñanza de idiomas en los centros educativos. Por otra, porque la inmersión dual, cuando se dirige a niños con trasfondo migrante, está orientada a fomentar un bilingüismo equilibrado desde la infancia y que estos puedan desempeñarse en ambas lenguas cuando sean adultos. De hecho, el bilingüismo es un indicador que se correlaciona positivamente con la presencia de estos hablantes en la enseñanza postobligatoria. Santibañez y Zárate (2014: 227-228) han concluido que los estudiantes

${ }^{4}$ En el curso 2019-2020, 121 escuelas en Utah disponían de programas de inmersión dual en español, 76 en chino, 32 en francés, 13 en portugués, dos en alemán y una en ruso (Watzinger-Tharp et alii, 2021: 200). 
La enseñanza de español como lengua de herencia en Estados Unidos: razones socioeconómicas y educativas que justifican su docencia universitaria en la década de 2020 | E. Garabaya Casado

bilingües que utilizan regularmente la lengua minoritaria aumentan sus posibilidades de acceso a la educación superior. Además, el éxito académico logrado gracias a la retención y al empleo de las lenguas de herencia en las escuelas beneficia especialmente a estas minorías para que alcancen el nivel universitario.

\subsection{La consolidación del campo de lenguas de herencia}

Con todo, podríamos pensar que, en la medida en que se retenga el bilingüismo en la infancia a partir de la escolarización dual, menos necesaria será la instrucción de lenguas de herencia cuando estos crezcan. Sin embargo, el hecho de que más de la mitad de los estados hayan aprobado desde finales del siglo pasado varias leyes de corte "English Only» (v. Crawford, 2008) nos obliga a mantenernos alerta. También preocupa el hecho de que, como advierten algunos autores como Valdez et alii (2016), el prestigio de la inmersión dual está promoviendo un proceso de gentrificación por el que las familias de habla inglesa están desplazando a los niños de etnias minoritarias de unos programas bilingües inicialmente diseñados para ellos.

Independientemente del modelo escogido, las estimaciones indican una gran presencia de niños latinos en las escuelas a medio y largo plazo. Solo en la actualidad, uno de cada cuatro niños en las aulas ya es de origen hispano (Jiménez, 2019: 151). A pesar de los esfuerzos por institucionalizar e incorporar las lenguas extranjeras dentro de los currículos escolares de todo el país, la realidad socioeconómica sugiere que precisamente los hijos de las etnias minoritarias tenderán a ser los principales candidatos a quedarse fuera, especialmente en el caso de las lenguas aún menos representadas que el español.

Por uno u otro motivo, los cursos de herencia para adultos seguirán siendo relevantes durante los años venideros. Valdés (2000: 8) destaca cómo han sido los propios hablantes de herencia los que no resignan a perder las lenguas de su familia y deciden matricularse en clases de estos idiomas en institutos y universidades. La constante demanda permite que el campo se siga actualizando gracias a la labor académica e investigadora de sus docentes. Por ejemplo, propuestas pedagógicas más modernas como el translanguaging (v. García, 2009: cap. 3; cf. Lyster, 2019) o el Learning by Design (v. Zapata, 2018) ofrecen nuevas e interesantes vías que seguir explorando a lo largo de esta década.

Hasta ahora, el estudio de los rasgos característicos de los hablantes de herencia ha permitido establecer una pedagogía más efectiva y, como resultado, la publicación de manuales que proporcionan una mejor formación a los profesores (p. ej., Beaudrie et alii, 2014; Fairclough y Beaudrie, 2016; Potowski, 2005; Zapata y Lacorte, 2018). Estas aportaciones resultan de suma importancia, ya que, como indican investigadores como Lacorte (2016), Potowski (2005), Said-Mohand (2013) o Schwartz (2001), los encargados de instruir un curso de herencia deben tener no solo conocimientos de la lengua española, sino también una buena base en sociolingüística, psicolingüística y en los procesos de adquisición de primeras y segundas lenguas, así como constancia de aquellos elementos socioculturales que determinan el trasfondo de estos estudiantes. 
La enseñanza de español como lengua de herencia en Estados Unidos: razones socioeconómicas y educativas que justifican su docencia universitaria en la década de 2020 | E. Garabaya Casado

La bonanza económica a la que nos referíamos en el anterior apartado encuentra un nítido reflejo en el ámbito educativo. En las últimas dos décadas, el porcentaje de hispanos con títulos universitarios, tanto de grado (Bachelor's Degree) como superiores se ha duplicado (Joint Economic Committee, 2020: 7). Además, los estudiantes de herencia ahora representan una proporción demográfica importante dentro de los programas de lenguas del país, y continuarán haciéndolo en las próximas décadas de acuerdo con las tendencias actuales (Carreira y Kagan, 2018: 154).

\section{CONCLUSIÓN}

Cada cierto tiempo, toda pedagogía debe someterse a un proceso de introspección para evaluar sus progresos. La nueva década, que se ha abierto camino entre la conmoción causada por la pandemia del coronavirus, será un escenario propicio para comprobar en qué medida triunfan o fracasan los empeños recientes por promover el bilingüismo en Estados Unidos y, con él, la enseñanza de las lenguas de herencia.

De momento, las cifras son claramente alentadoras. El aumento demográfico sostenido de la población hispana durante la próxima mitad del siglo augura que el español jugará un papel trascendental dentro de la sociedad estadounidense, ya sea como lengua primera, segunda o heredada. La mejora del estatus financiero de los latinos, aunque aún frágil ante las inclemencias socioeconómicas, animará a cada vez más jóvenes a optar por la vía universitaria y a labrarse unas mejores expectativas laborales. Una culminación del trayecto académico que será posible gracias a los programas bilingües encargados de facilitar el éxito escolar de las generaciones más jóvenes y tradicionalmente más vulnerables, frente a los currículos monolingües en los que partían desde una clara desventaja lingüística.

Por supuesto, la disposición y el arduo trabajo realizado por educadores e investigadores a lo largo de todo el país ha hecho posible que tengamos tantos motivos para el optimismo. Sus contribuciones en revistas, editoriales, conferencias y talleres han servido para concienciar al sector educativo de la existencia de una disciplina lingüística que merece la pena ser explorada con más detenimiento. Ser profesor de herencia no es solo una posibilidad vocacional para filólogos y sociolingüistas, sino una verdadera necesidad ante la demanda de tantos miles de alumnos que se presentan en las facultades del país al principio de cada curso académico y que lo seguirán haciendo durante este decenio.

No obstante, nos gustaría señalar que no es nuestra intención reducir la pedagogía de las lenguas minoritarias a una mera salida profesional con buenas expectativas laborales. El instructor de herencia debe disponer de voluntad de cambio, sacrificio, empatía y espíritu crítico para poner en práctica un tipo de didáctica contestataria, dirigida a desafiar muchas de las estructuras de poder establecidas. El objetivo último de los cursos para hablantes de herencia ha de ser el de promover su competencia en español como un medio para desarrollar a los estudiantes como adultos independientes, empoderados, bilingües y biculturales, capaces de contribuir y aportar valor humano a la sociedad en la que viven. 
La enseñanza de español como lengua de herencia en Estados Unidos: razones socioeconómicas y educativas que justifican su docencia universitaria en la década de 2020 | E. Garabaya Casado

\section{REFERENCIAS BIBLIOGRÁFICAS}

Acosta CoRTE, Álvaro (2013): «La adquisición y el desarrollo lingüístico de los hablantes de herencia de español: Un estudio de caso basado en la investigación-acción en el aula", Revista Nebrija de Lingüística Aplicada, 13.

AGIRDAG, Orhan (2014): "The literal cost of language assimilation for the children of immigration: The effects of bilingualism on labor market outcomes", en Rebecca. M. CALLAHAN y Patricia C. GÁndARA, eds., The Bilingual Advantage: Language, Literacy, and the U.S. Labor Market, Bristol, Multilingual Matters, 160-181.

BAKER, Colin y Wayne E. WriGHT (2017): Foundations of Bilingual Education and Bilingualism, 6. ${ }^{\text {a }}$ ed., Bristol, Multilingual Matters.

BeAudRiE, Sara, Cynthia Ducar y Kim Potowski, eds. (2014): Heritage language teaching: Research and practice, Columbus, McGrawHill Education.

BLAKE, Robert (1983): «Mood selection among Spanish-speaking children, ages 4 to 12", The Bilingual Review/La Revista Bilingüe, 10, 1, 2132, https://www.jstor.org/stable/25744036.

CARREIRA, María M. (2007): «El español a la venta: la lengua en el mercado global», en Manuel LACORTE, coord., Lingüística aplicada del español, Madrid, Arco, 521-548.

CARREIRA, María M. y Regla Armengol (2001): «Professional Opportunities for Heritage Language Speakers», en Joy Kreeft PEYTON, Donald A. RANARD y Scott McGINNIS, eds., Heritage Languages in America: Preserving a National Resource, McHenry, Delta Systems Inc., 109-142.

Carreira, María M. y Olga Kagan (2011): «The Results of the National Heritage Language Survey: Implications for Teaching, Curriculum Design, and Professional Development», Foreign Language Annals, 44, 1, 40-64, https://doi.org/10.1111/i.19449720.2010.01118.x.

CARREIRA, María M. y Olga Kagan (2018): «Heritage language education: A proposal for the next 50 years», Foreign Language Annals, 51, 152168, https://doi.org/10.1111/flan.12331.

Collier, Virginia P. y Wayne P. ThOMAs (2007): "Predicting Second Language Academic Success in English Using the Prism Model», en
J. CUMmins y C. DAvison, eds., International Handbook of English Language Teaching, Boston, Springer Science and Business Media, 333-348.

CRAWFORD, James (2008, 24 junio): «Language Legislation in the U.S.A.» languagepolicy.net, [en línea]: $<$ http://www.languagepolicy.net/archives/langl eg.htm\#State>. [Consulta: 27/06/2021].

DıCKER, Sue J. (2000): «Official English and Bilingual Education: The Controversy over Language Pluralism in U.S. Society», en Joan Kelly HaLL y William G. EGGINGTON, eds., The Sociopolitics of English Language Teaching, Clevedon/Búfalo, Multilingual Matters, 45-66.

FAIRClough, Marta Ana y Sara BeAUdRIE, eds. (2016): Innovative strategies for heritage language teaching: A practical guide for the classroom, Washington, DC, Georgetown University Press.

FISHMAN, Joshua A. (2001): «300-plus Years of Heritage Language Education in the United States", en Joy Kreeft PEYTON, Donald A. RANARD y Scott McGinNIs, eds., Heritage Languages in America: Preserving a National Resource, McHenry, Delta Systems Inc., 8197.

FuLLER, Janet M. y Jennifer LEEMAN (2020): Speaking Spanish in the US. The Sociopolitics of Language, Bristol, Multilingual Matters, 2. ${ }^{a}$ ed., https://doi.org/10.21832/FULLER8281.

GARCíA, Ofelia (2009): Bilingual Education in the $21^{\text {st }}$ Century: A Global Perspective, Malden, WileyBlackwell.

Hudgens Henderson, Mary, Damián Vergara WILSON y Michael R. WOODS (2020): «How Course Level, Gender, and Ethnic Identity Labels Interact with Language Attitudes towards Spanish as a Heritage Language», Hispania, 103, 1, 27-42, https://doi.org/10.1353/hpn.2020.0008.

InSTITUTO CERVANTES (2020): El español: una lengua viva. Informe 2020, [en línea]: $<$ https://cvc.cervantes.es/lengua/espanol_len gua_viva/pdf/espanol_lengua_viva_2020.pdf>. [Consulta: 27/06/2021].

JIMÉNEZ, Juan Carlos (2019): «El valor económico del español en Estados Unidos», Boletín Económico del ICE, 3110, 141-156, 
La enseñanza de español como lengua de herencia en Estados Unidos: razones socioeconómicas y educativas que justifican su docencia universitaria en la década de 2020 | E. Garabaya Casado

https://doi.org/10.32796/bice.2019.3110.679 4.

JOINT ECONOMIC COMMITTEE (2020): «The Economic State of the Hispanic Community in America: Keys to Building a Better Economy after COVID-19». U. S. Congress, 30 de sept. [en línea]:

$<$ https://www.jec.senate.gov/public/index.cfm /democrats/2020/9/the-economic-state-ofthe-hispanic-community-in-america>. [Consulta: 27/06/2021].

LACORTE, Manel (2016): «Teacher Development in Heritage Language Education", en Marta FAIRCLOUGH y Sara BEAUDRIE, eds., Innovative strategies for heritage language teaching: $A$ practical guide for the classroom, Washington, DC, Georgetown University Press, 99-119.

LIPSKI, John M. (2003): «La lengua española en los Estados Unidos: Avanza a la vez que retrocede», Revista Española de Lingüística, 33, 2, 231-260.

LIPSKI, John M. (2015): «Dialectos del español de América: los Estados Unidos", en Javier GutiéRREZ REXACH, coord., Enciclopedia de lingüística hispánica, Londres, Routledge, 2, 363-374.

LoPEZ, Mark Hugo, Lee RAINIE y Abby Budiman (2020): «Financial and health impacts of COVID-19 vary widely by race and ethnicity", Pew Research Center, 5 de mayo, [en línea]: $<$ https://www.pewresearch.org/facttank/2020/05/05/financial-and-healthimpacts-of-covid-19-vary-widely-by-race-andethnicity/>. [Consulta: 27/06/2021].

LYSTER, Roy (2019): «Translanguaging in Immersion: Cognitive Support or Social Prestige?», The Canadian Modern Language Review, 75, 4, 340352, https://doi.org/10.3138/cmlr.2019-0038.

MenKen, Kate (2010): «No Child Left Behind and English language learners: The challenges and consequences of high-stakes testing», Theory Into Practice, 49 ,

121-128, https://doi.org/10.1080/00405841003626619.

MONTRUL, Silvina (2002): «Incomplete acquisition and attrition of Spanish tense/aspect distinctions in adult bilinguals», Bilingualism: Language and Cognition, 5, 1, 39-68, https://doi.org/10.1017/S1366728902000135.

MoNTRUL, Silvina (2008): Incomplete acquisition in bilingualism. Re-examining the age factor, Ámsterdam/Filadelfia, John Benjamins Publishing Co.

MORENO FERNÁNDEZ, Francisco (2008): «Dialectología hispánica en los Estados Unidos», en Humberto LóPEZ MORALES, coord., Enciclopedia del español en los Estados
Unidos. Anuario del Instituto Cervantes 2008, Madrid, Instituto Cervantes/Santillana.

PотоWsKI, Kim (2005): Fundamentos de la enseñanza del español a hispanohablantes en los EE. UU., Madrid, Arco.

POTOWSKI, Kim, Jill JEGERSKI y Kara MORGAN-SHORT (2009): «The Effects of Instruction on Linguistic Development in Spanish Heritage Language Speakers", Language Learning, 59, 3, 537579, $\quad$ https://doi.org/10.1111//.14679922.2009.00517.x.

SAID-MohAND, Aixa (2013): «El español como lengua de herencia en los EE. UU.: retos para su didáctica», MarcoELE, revista didáctica, 16, [en línea]: $<$ https://marcoele.com/descargas/16/saidmohand-lengua_de_herencia.pdf>. [Consulta: 27/06/2021].

SANTIBAÑEZ, Lucrecia y Maria Estela ZÁRATE (2014): «Bilinguals in the US and College Enrollment», en Rebecca. M. Callahan y Patricia C. GÁnDARA, eds., The Bilingual Advantage: Language, Literacy, and the U.S. Labor Market, Bristol, Multilingual Matters, 211-233.

SILVA-CORVALÁN, Carmen (2012): "Acquisition of Spanish in Bilingual Contexts», en José Ignacio Hualde, Antxon OlarRea y Erin O'Rourke, eds., The Handbook of Hispanic Linguistics, Malden, Wiley-Blackwell, 783-801.

SILVA-CORVALÁN, Carmen (2014): «La adquisición del español en niños de tercera generación", Informes del Observatorio/Observatorio Reports, [en línea]: $<$ http://cervantesobservatorio.fas.harvard.edu/ sites/default/files/005 informes csc adquisici on_espanol_tercera_generacion.pdf>. [Consulta: 27/06/2021].

SILVA-CORVALÁN, Carmen (2018): "Simultaneous bilingualism: Early developments, incomplete later outcomes?», International Journal of Bilingualism, 22, 5, 497-512.

SCHWARTZ, Ana María (2001): «Preparing Teachers to Work With Heritage Language Learners", en Joy Kreeft Peyton, Donald A. RanaRd y Scott McGINNIS, eds., Heritage Languages in America: Preserving a National Resource, McHenry, Delta Systems Inc., 229-252.

U. S. Census Bureau (2020): «Table 2. Population by Sex, Age, and Hispanic Origin Type: 2019», [en línea]: $<$ https://www2.census.gov/programssurveys/demo/tables/hispanicorigin/2019/2019-cps/cps-2019-hispanictab2.xlsx>. [Consulta: 27/06/2021]. 
La enseñanza de español como lengua de herencia en Estados Unidos: razones socioeconómicas y educativas que justifican su docencia universitaria en la década de 2020 | E. Garabaya Casado

VALDÉS, Guadalupe (2000): «Introduction», en N. ANDERSON, ed., Spanish for native speakers: American Association of Teachers of Spanish and Portuguese professional development series handbook for teachers K-16, Nueva York, Harcourt, 1, 1-20.

VALDÉS, Guadalupe (2001): «Heritage Language Students: Profiles and Possibilities», en Joy Kreeft PeYton, Donald A. Ranard y Scott McGINNIS, eds., Heritage Languages in America: Preserving a National Resource, McHenry, Delta Systems Inc., 37-77.

VALDEZ, Verónica E., Juan A. FreIRE y M. Garrett DeLAVAN (2016): «The Gentrification of Dual Language Education", Urban Rev, 48, 601627, https://doi.org/10.1007/s11256-0160370-0.

Van Deusen-Scholl, Nelleke (2003): «Toward a Definition of Heritage Language: Sociopolitical and Pedagogical Considerations", Journal of Language, Identity, and Education, 2, 3, 211230, https://doi.org/10.1207/S15327701JLIE0203 4.

WATZINGER-THARP, Johanna, Douglas S. THARP y Fernando RuBIO (2021): «Sustaining Dual
Language Immersion: Partner Language Outcomes in a Statewide Program», The Modern Language Journal, 105, 1, 194-217, https://doiorg.ezproxy.lib.utah.edu/10.1111/modl.12694

WONG FILLMORE, Lily (1991): "When Learning a Second Language Means Losing the First", Early Childhood Research Quarterly, 6, 323346, $\quad$ https://doi.org/10.1016/S08852006(05)80059-6.

WriGHT, Wayne E. (2019): Foundations for Teaching English Language Learners: Research, Theory, Policy, and Practice, Filadelfia, Caslon, 3 . $^{\mathrm{a}}$ ed.

ZAPATA, Gabriela C. (2018): «A Match Made in Heaven: An Introduction to Learning by Design and Its Role in Heritage Language Education», en Gabriela C. ZAPATA y Manel LACORTE, eds., Multiliteracies Pedagogy and Language Learning: Teaching Spanish to Heritage Speakers, Cham, Palgrave Macmillan, 1-26.

ZAPATA, Gabriela C. y Manel LACORTE, eds. (2018): Multiliteracies Pedagogy and Language Learning: Teaching Spanish to Heritage Speakers, Cham, Palgrave Macmillan. 